\title{
Optimal Two-Stage Filtering of Elastograms
}

\author{
Suba R. Subramaniam, Student Member, IEEE, EMBS, Tsz K. Hon, Student Member, IEEE, EMBS, \\ Wing-Kuen Ling, Member, IEEE, and Apostolos Georgakis, Member, IEEE.
}

\begin{abstract}
In ultrasound elastography, tissue axial strains are obtained through the differentiation of measured axial displacements. However, during the measurement process, the displacement signals are often contaminated with decorrelation noise caused by changes in the speckle pattern in the tissue. Thus, the application of the gradient operator on the displacement signals results in the presence of amplified noise in the axial strains, which severely obscures the useful information. The use of an effective denoising scheme is therefore imperative. In this paper, a method based on a twostage consecutive filtering approach is proposed for the accurate estimation of axial strains. The presented method considers a cascaded system of a frequency filter and a time window, which are both designed such that the overall system operates optimally as a minimum variance estimator. Experimentation on simulated signals shows that the two-stage scheme employed in this study has good potential as a denoising method for ultrasound elastograms.
\end{abstract}

\section{INTRODUCTION}

$\mathrm{U}$ ltrasound elastography is a relatively new medical technique which can provide images of the strain distribution of soft tissue under static compression [1]. It is most commonly used to reveal changes in the tissue stiffness which may be due to abnormal pathological processes, such as cancer. A strain image is obtained by reconstructing the mechanical properties of the tissue based on measurements of internal deformations when small amounts of external compression are applied to the tissue. The exerted compression induces a displacement of the non-uniformities in the tissue which can cause scattering of the ultrasound wave. Thus, by comparing ultrasound readings before and after compression the induced displacement of the tissue can be determined [2]. Finally, the strains are calculated as the first-order derivatives of the measured displacements. The resulting strain matrix which is typically displayed as a grey scale image is called the elastogram.

Unfortunately, the spatial distribution of the tissue scatterers used for displacement tracking undergoes changes under the applied compression (speckle pattern decorrelation) and thus, the measured displacements become corrupted with noise. Although the statistics of this noise are unknown, the desired signal consists largely of low frequencies apart from the areas of the boundary between the

\section{Manuscript received April 14, 2011}

S. R. Subramaniam (e-mail: suba.r.subramaniam@kcl.ac.uk), Tsz K Hon (e-mail: tsz_kin.hon@kcl.ac.uk), and A. Georgakis (tel: 0044207848 1101; fax: 0044207848 2932; e-mail: apostolos.georgakis@kcl.ac.uk) are with the Division of Engineering, King's College London, London WC2R 2LS, UK. Wing-Kuen Ling (email: wling@ lincoln.ac.uk) is with the School of Engineering, University of Lincoln, Lincoln, LN6 7TS, UK. (healthy) medium and the (potentially malicious) inclusion, where relatively higher frequencies are present. Since the gradient operator is known to boost high frequencies the noise components mainly dominate the resulting elastogram. Denoising of the elastogram is clearly necessary; however, the application of a conventional low-pass filter is problematic due to the global effect of the cut-off frequency on the signal. If the cut-off threshold is set too low then noise will be eliminated at the expense of a loss of resolution around the boundary of the inclusion. On the other hand, if the cut-off is set higher then undesirable amounts of noise will also pass through. Therefore, any scheme based on a single filter would be unable to denoise elastography signals effectively.

Alternative denoising methods such as those based on discrete wavelet transforms (WT) [3], [4], fractional Fourier transform (FrFT) [5] and short-time Fourier transform (STFT) [6] have been proposed for the accurate estimation of strain signals. Despite the preliminary nature of the above works, the presented results appeared promising. However, a number of limitations seem to be inherent in those approaches. For example, the efficiency of wavelet denoising heavily relies on a number of factors such as the choice of basis functions, the number of decomposition levels, and the thresholding strategy. Even worse, the truncation of wavelet coefficients often generates interference in the form of pseudo-Gibbs artifacts. Meanwhile, the efficiency of methods based on the FrFT and STFT - at their current phase - also depends on empiricallydetermined parameters.

The method proposed here is based upon the idea of filtering consecutively in different domains [7]. Such an operation was shown to potentially outperform any singlestage filtering approach. In this work, a two-stage denoising system is employed which consists of a Fourier-domain filter followed by a time-domain window. Following the method in [7] both the frequency response of the filter and the shape of the time window are designed such that the overall system yields an optimal result in a minimum variance sense. We further compare the performance of the proposed method with that of a single-stage optimal filter as well as with the STFT and FrFT-based approaches using simulated elastograms.

Section II provides a concise overview of the theoretical background and describes the algorithm. In section III, experimental results are provided. Conclusions are finally drawn in section IV. 


\section{THEORETICAL BACKGROUND}

\section{A. Optimal frequency-domain filter}

In measurements under additive noise, the following observation model can be assumed in discrete form:

$$
y=x+n,
$$

where $y, \boldsymbol{x}, \boldsymbol{n}$ are column vectors of size $N$ representing the acquired signal, the ideal process, and the noise realisation, respectively. The goal of the filter is to find an estimate, $\mathbf{s}$, which would be as close as possible to the ideal $\boldsymbol{x}$. A natural optimality criterion is the mean square error (MSE), which can be defined as:

$$
\operatorname{mse}(\mathrm{x})=\frac{1}{\mathrm{~N}} \mathrm{E}\left[\mathrm{s}-\left.\mathrm{x}\right|^{2}\right],
$$

which, due to Parseval's relation is also equal to:

$$
\operatorname{mse}(\hat{\mathbf{X}})=\frac{1}{\mathrm{~N}} \mathrm{E}\left[|\hat{\mathbf{X}}-\mathbf{X}|^{2}\right]
$$

where $\hat{\mathbf{x}}$ is the Fourier transform of $\hat{\mathbf{s}}$, i.e. $\hat{\mathbf{X}}=\vec{F} \mathrm{~s}$, with $F$ being the DFT matrix. For the common setting depicted in Fig.1a it holds that:

$$
\mathrm{X}=A Y
$$

where $\mathbf{Y}=F y$, and $A$ is a diagonal matrix whose non-zero elements $\left(h_{0}, h_{1}, \ldots, h_{N-1}\right)$ form the frequency response of the filter. By minimising (3) with respect to $\left(h_{0}, h_{1}, \ldots, h_{N-1}\right)$ the $N$ components of the optimal frequency response can be determined as:

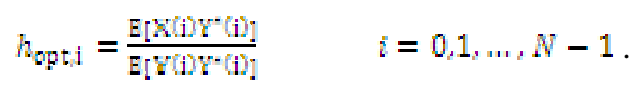

Under the assumption that the noise is independent of the ideal signal, (5) can be expressed as:

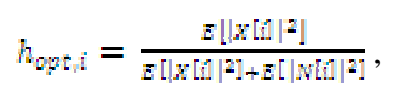

which is the ensemble average energy density of the signal at the time instant $i$, divided by the sum of the ensemble averages of the energy densities of the signal and noise at the same time instant.

\section{B. Optimal two-stage filtering system}

Considering the system configuration shown in Fig. 1b, the estimate $\mathbf{s}$ is equal to:

$$
\mathrm{s}=A_{2} F A_{1} F y,
$$

where

$$
A_{k}=\left[\begin{array}{ccccc}
h_{h / 1} & 0 & \cdots & 0 & 0 \\
0 & & & & 0 \\
\vdots & & \cdots & & \vdots \\
0 & & & & 0 \\
0 & 0 & \cdots & 0 & h_{k N}
\end{array}\right], \quad k=1,2
$$

with $h_{k}=\operatorname{diag}\left(A_{k 2}\right), \quad$ i.e. $\quad h_{1}=\left(h_{1,0}, h_{1,1}, \ldots, h_{1, N-1}\right) \quad$ and $h_{2}=\left(h_{2,0}, h_{2,1}, \ldots, h_{2, N-1}\right)$, being the frequency response of the filter and the window function, respectively. The objective is then to determine the optimal $h_{1}$ and $h_{2}$ which minimise
(2). The non-linearity of the problem makes it difficult to

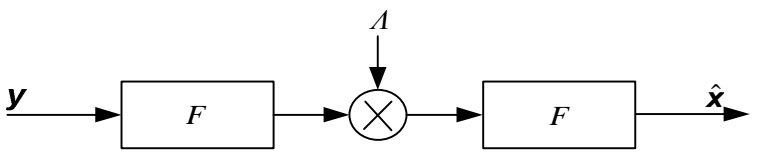

(a)

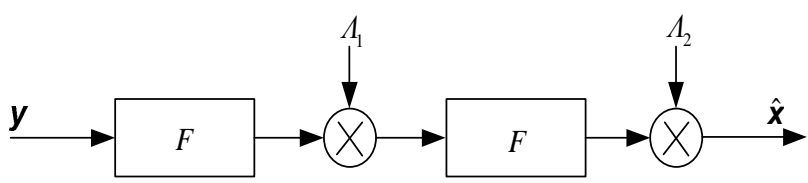

(b)

Fig. 1 (a) Block diagram of a single stage filter, (b) Block diagram of the two-stage filtering approach.

find an analytic solution. A usual approach in such optimisation problems is to adopt an iterative procedure in which one function is optimised at a time by keeping the other fixed to its values obtained during the preceding iteration. Such an approach has also been followed in [7] and is briefly summarised in the following paragraphs.

At the beginning, both the diagonal matrices $A_{1}$ and $A_{2}$ are initialized to identity matrices. Then, starting with the first function $h_{1}$, an estimate for its optimum expression can be calculated based on (5).

In the second iteration, the preliminary solution for $h_{1}$ is used to obtain an initial estimate of the optimum $h_{2}$. Thus, (7) can be written as $\hat{x}=A A_{2} B y$, where $A=I$ (the identity matrix) and $B=F A_{1} F$. Minimising (2) yields [7]:

$$
h_{2}=D^{-1} c \text {, }
$$

where $D=\left(A^{H} A\right) *\left(B R_{y} B^{H}\right)^{T}$, and '*' denotes the element-wise multiplication between two matrices, whereas $c=\operatorname{diag}\left(A^{H} R_{x y} B^{H}\right)$. Also, $R_{x y}=E\left[x y^{H}\right] \quad$ and $R_{y y}=E\left[y y^{M}\right]$ are correlation matrices, which under the assumption of the noise being independent of the ideal signal can also be obtained as $R_{x y}=R_{x x}$ and $R_{y y}=R_{x x}+R_{n n}$.

The third iteration focuses back on $h_{1}$ with (7) now expressed as $\hat{\mathbf{x}}=A A_{1} E y$, where $A=A_{2} F$ and $B=F$. By minimising (2) the solution is obtained similarly to (8), i.e. $h_{1}=D^{-1} c$, with $D$ and $c$ having the same structure as before.

The above steps are repeated with $h_{1}$ and $h_{2}$ being updated accordingly at each iteration. Once the solutions converge the iterations stop.

\section{Best-case scenarios}

The motivation for investigating the above two-stage filtering scheme as a possible method for enhancing ultrasound elastograms arises from the performance advantages that this approach may have over a simple single-stage filter. To illustrate this, we compare the bestcase scenarios for both filters, i.e. assuming that the statistics of the ideal signal and the noise are known, we examine 
what is the optimal result that can be achieved by each of the two methods.

Fig. 2a depicts a noise-free (ideal) simulated elastogram, and Fig. $2 b$ shows a realization of the corrupted elastogram. The denoised result of the single-stage optimal filter is presented in Fig. 2c, whereas the result based on the application of the two-stage scheme is shown in Fig. 2d. It is clear that the second method yields a much more accurate estimate of the ideal elastogram.

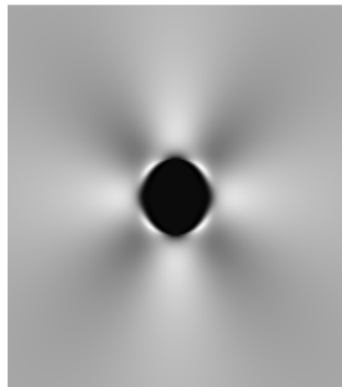

(a)

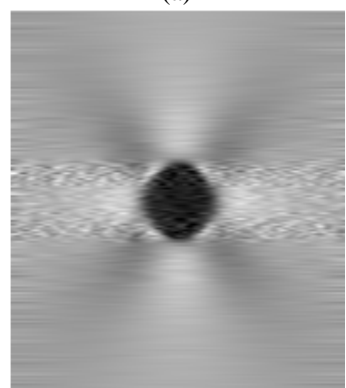

(c)

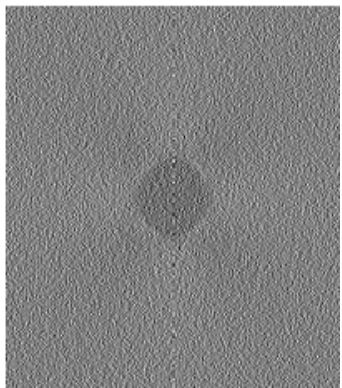

(b)

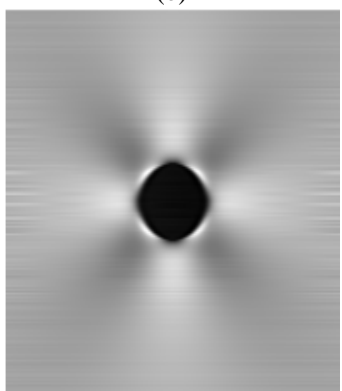

(d)
Fig. 2. (a) Simulation of ideal elastogram, and (b) corrupted elastogram $(\mathrm{SNR}=-16.28 \mathrm{~dB})$. Recovered elastogram after denoising with: (c) the single-stage optimal filter, and (d) the optimal two-stage system.

\section{EXPERIMENTAL RESULTS}

The tissue displacement data that was used in our experiments was simulated using the two-dimensional (2-D) analytic model equations introduced by Muskhelishvili [8]. An ideal mechanical strain image using this model is depicted in Fig. 2a. The model assumes that the tissue was subjected to an inward uniaxial compression of $314 \mathrm{~Pa}$, under the condition that the strain is minimal in the outward direction of the plane (plane-strain state). The dimension of the simulated phantom was $100 \times 100 \mathrm{~mm}$ with an inclusion radius of $10 \mathrm{~mm}$ which is assumed to be 4 times stiffer than the background medium.

Zero-mean white Gaussian noise was added to the displacement at a signal-to-noise (SNR) ratio of $40 \mathrm{~dB}$. The axial strain was computed using first-order forward differences of the noisy displacement image along the horizontal direction. The resulted elastogram has an SNR of - 16.28 dB. Fig. 3a shows the central slice of a noisy realization of the displacement while Fig. $3 \mathrm{~b}$ presents the corresponding central slice of the elastogram. The severe amplification of the noise due to the differentiation process and the consequent distortion of the elastogram can be appreciated.

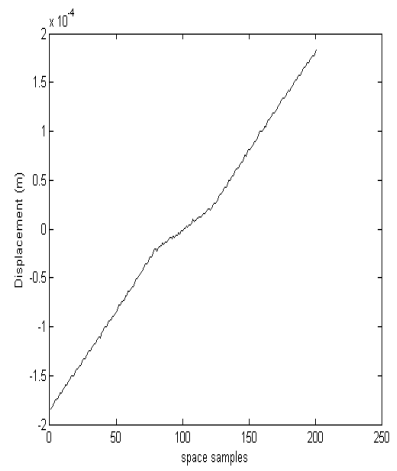

(a)

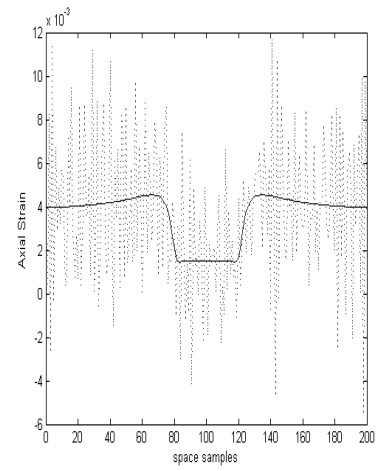

(b)
Fig. 3. (a) Noisy displacement profile taken along the center of the displacement image (b) Calculated axial strain slice (dotted line) taken along the center of the resulting elastogram contrasted to the ideal axial strain (solid line).

The contrast-to-noise ratio $\left(\mathrm{CNR}_{\mathrm{e}}\right)$ proposed in [9] was used in our experiments to quantify the performance of the different methods and facilitate comparisons between them. $\mathrm{CNR}_{\mathrm{e}}$ is defined as:

$$
\mathrm{CNR}_{\mathrm{E}}(\mathrm{dB})=20 \log _{10}\left(\frac{2 u_{x+1}-H_{52}{ }^{2}}{\left(\sigma_{31}^{2}+\sigma_{22}^{2}\right)}\right)
$$

where $\mu_{\mathrm{s} 1}$ and $\mu_{\mathrm{s} 2}$ represent the mean value of strain in the

inclusion and the medium, and $\sigma_{\mathrm{s} 1}$ and $\sigma_{\mathrm{s} 2}$ denote the strain

variances, respectively. A high $\mathrm{CNR}_{\mathrm{e}}$ signifies a readable elastogram whereas low values of this metric indicate a poor image.

Along with the single-stage Fourier filter and the twostage filtering system described in Section II, two other recently proposed methods have been included in the conducted experiments. The FrFT-based denoising method [5] and the masked STFT scheme described in [6] were applied to the same noisy realization of the simulated elastogram that was used for all the presented results. The parameters required for the latter two methods were determined empirically as this was done in [5] and [6], respectively. For the given realization the FrFT-based method achieved a $\mathrm{CNR}_{\mathrm{e}}$ value of $65.69 \mathrm{~dB}$ whereas the masked STFT approach resulted in a value of $60.60 \mathrm{~dB}$. The resulting elastograms are shown in Fig.4c and Fig.4d, while the axial strains corresponding to the central slices of the filtered elastograms are compared in Fig.5c and Fig.5d.

Both equations (6) and (8) refer to averages obtained from ensembles of realizations of the ideal and noise processes. In a real-world experiment these would not be available and therefore need to be estimated. In this work, we treated the 
simulated elastogram as an experimental measurement therefore assuming that the ideal signal is unknown. Instead, we generated a number of estimates of the ideal process by low-pass filtering simulated noisy elastograms at different cut-off frequencies. The simulated noisy elastograms could represent repeated ultrasonic measurements of the same tissue area under varied compression levels. The noise ensemble was created by generating a number of noise realizations and then taking their first derivative. In the clinical lab, this could be replaced by a few measurements of healthy tissue displacements, which would then be differentiated with their offset being subsequently removed.

Based on the above sets, estimates of the correlations $R_{z:}$ and $R_{n n}$ were obtained and used for the derivation of the multiplicative functions of the methods in Section II. The proposed two-stage filter resulted in a $\mathrm{CNR}_{\mathrm{e}}$ value of $66.93 \mathrm{~dB}$ whereas the single-stage filter achieved a lower ratio of $49.08 \mathrm{~dB}$. The resulting elastograms are shown in Fig.4a and Fig.4b. The central slices of the filtered elastograms are compared in Fig.5a and Fig.5b.

\section{DISCUSSION}

We have presented a new approach for enhancing ultrasound elastograms. The method is based on a two-stage system comprising two multiplicative functions of which one is applied in the frequency domain and the other in the time-domain. The overall system was designed as a minimum variance estimator and the two functions were optimized accordingly. Using simulated data we explored the potential advantages of this method in denoising elastograms in comparison with Fourier filtering as well as with two more modern approaches based on the STFT and the FrFT. The experimental outcomes indicate that the method can outperform the other schemes with respect to the achieved $\mathrm{CNR}_{\mathrm{e}}$ values, and also according to what visual inspection of the resulting elastograms suggests. It should be noted that each of the two modern approaches included in our comparisons had independently been shown [5], [6] to be superior to commonly used denoising approaches for the elastogram. Further experimentation involving clinical data will have to follow in order to validate this new approach and fully assess its advantages and limitations.

\section{REFERENCES}

[1] J. Ophir, I. Cespedes, H. Ponnekanti, Y. Yazdi and X.Li, "Elastography: A quantitative method for imaging the elasticity of biological tissues", Ultrasonic Imaging, vol. 13, pp. 111-134, 1991.

[2] M. O'Donnell, A.R. Skovoroda, B.M. Shapo and S.Y.Emelianov, "Internal displacement and strain imaging using ultrasonic speckle tracking", IEEE Trans. Ultrason. Ferroelect. Freq. Contr., vol. 41, no. 3, pp. 314-325, 1994.

[3] U. Techavipoo and T. Varghese, "Wavelet denoising of displacement estimates in elastography", Ultrasound. Med. \& Biol., vol. 30, no. 4 pp. 477-491, 2004.

[4] L. Xu, J. C. Bamber, and D. MelodeLima, "Wavelet Shrinkage-Based Strain Estimation for Elastography", IEEE Ultrasonics Symposium, Rotterdam, the Netherlands, 2005.

[5] S. R. Subramaniam, T. K. Hon, A. Georgakis, and G. Papadakis, "Fractional Fourier-Based Filter for Denoising Elastograms", in Proc. IEEE Engineering in Medicine and Biology (EMBC), Buenos Aires, Argentina, 2010.

[6] T. K. Hon, S. R. Subramaniam, A. Georgakis, and S. Alty, "STFTbased denoising of elastograms", in Proc. IEEE Int. Conf. Acoust., Speech Signal Process. (ICASSP), Prague, Czech Republic, 2011.

[7] M.F. Erden, M.A. Kutay, and H.M. Ozaktas, "Repeated filtering in consecutive Fractional Fourier Domains and Its Application to Signal Restoration”, IEEE Trans. Sig. Proc., vol. 47, pp. 1458-1462, 1999.

[8] Muskhelishvili NI. Some Basic Problems of the Mathematical Theory of Elasticity. Groningen, The Netherlands: Noordhoff, 1963 (transl. from Russian by JRM Radok); Chap. 9; pp. 216-223.

[9] Varghese T, Ophir J. "An analysis of elastographic contrast-to-noise ratio performance", Ultrasound Med. \& Biol., vol.24, pp. 915-924, 1998.

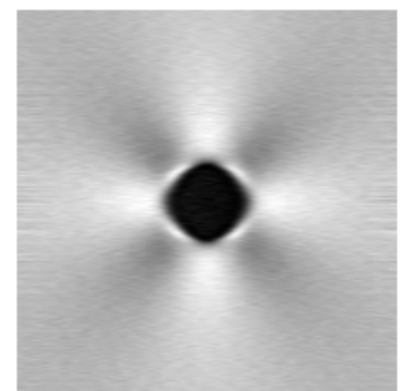

(a)

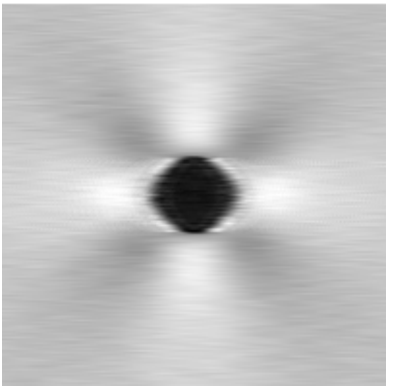

(c)

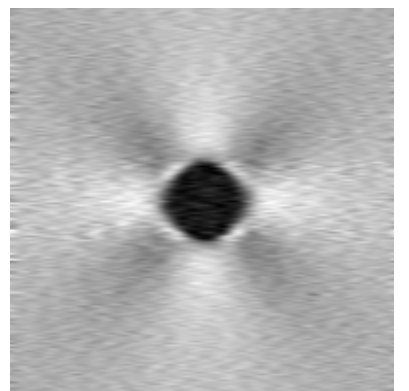

(b)

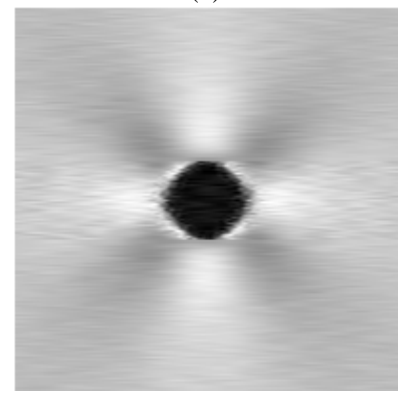

(d)

Fig. 4. Calculated elastogram after denoising with: (a) the proposed twostage filter, (b) the single-stage filter, (c) the FrFT-based filter, and (d) the masked STFT method.

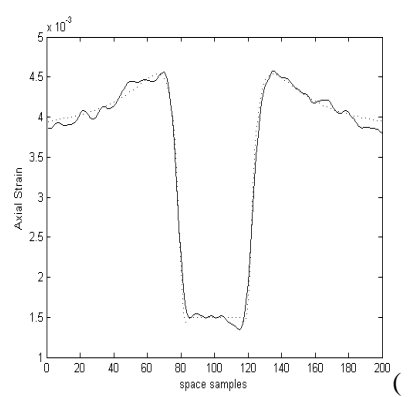

a)

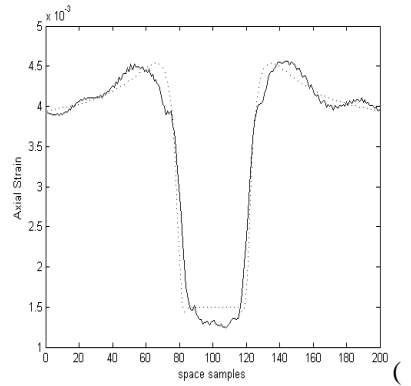

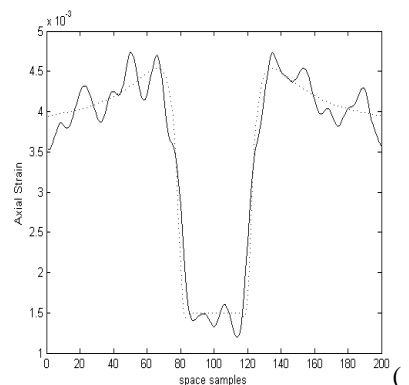

b)

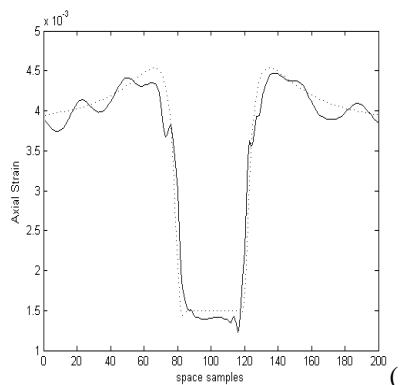


c)

d)

Fig. 5. Axial strain slices (solid lines) taken along the center of the resulting elastogram after denoising with: (a) the proposed two-stage filter, (b) the single-stage filter, (c) the FrFT-based filter, and (d) the masked STFT

method. The reference axial strain is also shown (dotted line). 